\title{
Belgeo
}

\section{Éditorial : Art(s) \& Espace(s) / Art(s) \& Space(s)}

\section{Camille Boichot, Tatiana Debroux et Boris Grésillon}

\section{(2) OpenEdition}

Journals

Édition électronique

URL : http://journals.openedition.org/belgeo/13398

ISSN : 2294-9135

Éditeur :

National Committee of Geography of Belgium, Société Royale Belge de Géographie

Référence électronique

Camille Boichot, Tatiana Debroux et Boris Grésillon, «Éditorial : Art(s) \& Espace(s) / Art(s) \& Space(s) », Belgeo [En ligne], 3 | 2014, mis en ligne le 19 décembre 2014, consulté le 22 septembre 2020. URL http://journals.openedition.org/belgeo/13398

Ce document a été généré automatiquement le 22 septembre 2020.

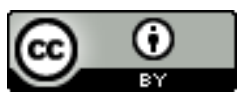

Belgeo est mis à disposition selon les termes de la licence Creative Commons Attribution 4.0 International. 


\title{
Éditorial : Art(s) \& Espace(s) / Art(s) \& Space(s)
}

\author{
Camille Boichot, Tatiana Debroux et Boris Grésillon
}

1 Pour qui s'intéresse à la géographie et aux propositions qui émanent de ses chercheurs anglophones ou francophones, cela n'a pu échapper : les événements et les publications qui mêlent approche spatiale, artistes et productions artistiques se suivent et ne se ressemblent pas.

2 Ce qui, il n'y a pas si longtemps, pouvait apparaître comme des pratiques et objets de recherche très marginaux, s'affirme désormais dans le paysage scientifique contemporain de la géographie sous la bannière encore mouvante de la géographie culturelle ou de la géographie de l'art ${ }^{1}$. En effet, les productions artistiques en tout genre, leurs acteurs, leurs enjeux et leurs lieux sont autant de portes d'entrées mobilisées par les géographes pour réinterroger l'espace, la production culturelle et celle du territoire, ou leur propre rapport au terrain. Le caractère aujourd'hui récurrent de sessions spécialement dédiées à ces problématiques lors des grandes rencontres internationales de la géographie ${ }^{2}$ renforce l'impression générée par les travaux et les publications de plus en plus nombreuses : il se passe décidément des choses qui fascinent les géographes à la frontière entre les arts et l'espace.

3 Forts de ce constat, mais conscients aussi du caractère encore marginal de ces travaux et de leur visibilité dans le champ de la géographie humaine, nous avons proposé l'idée d'un numéro thématique au comité éditorial de la revue Belgeo. Sept ans après la parution d'un numéro de la revue des TIGR consacré aux "Spatialités de l'Art " ${ }^{3}$, nous avons lancé un appel à textes qui proposait de prendre les acteurs, les pratiques et les manifestations artistiques comme objets d'analyse d'une géographie de l'art. Ce faisant, nous espérions rassembler différents regards sur les relations entre art(s) et espace(s), envisagées dans leur pluralité par des géographes et d'autres chercheurs en sciences humaines partageant ces réflexions. Nous avons invité les auteur(e)s à inscrire leurs propositions dans trois axes: le premier visait à rassembler des réflexions épistémologiques concernant le développement d'une géographie de l'art pour comprendre ses spécificités et ses apports (L'art comme objet de recherche en géographie), 
les deux autres envisageaient les spatialités artistiques selon deux modes complémentaires liés à la localisation (Les arts dans l'espace) et aux dynamiques spatiales (Mobilités artistiques) des acteurs et des phénomènes artistiques.

4 Nous n'avons pas été déçus. Sur près de quarante-cinq propositions d'articles, nous publions finalement dans ce numéro quinze textes qui témoignent de la richesse et de la grande diversité des travaux menés actuellement - tant sur le plan des objets (réseaux artistiques, street art, pratiques de terrain, équipements culturels, quartiers artistiques...), des espaces étudiés (villes occidentales, villes de pays émergents, villes "des suds») que des disciplines scientifiques des auteurs (géographes bien sûr, mais aussi urbanistes, historiens des arts ou sociologues).

5 "I speak here not as a geographer, but as an artist» (Reclus et al., 1903, p. 298, cité par Ferretti dans ce numéro). En citant ces quelques mots prononcés par Elisée Reclus à la Royal Geographical Society de Londres en 1903, Federico Ferretti nous propose d'emblée de réfléchir à la figure du géographe et à son rapport à l'art dans l'illustration de ses travaux, qu'il s'agisse de réalisations cartographiques, de modèles ou d'illustrations. Dans un article intitulé «La nature comme œuvre d'art: Elisée Reclus et les (néo)impressionnistes ", l'auteur s'intéresse non seulement aux réseaux de sociabilité du géographe anarchiste avec les artistes (néo)impressionnistes, qui influencèrent sa pensée, mais aussi à la conceptualisation reclusienne du monde comme une œuvre.

6 L'art serait à la fois un moyen de penser et un outil d'expression complémentaire pour le géographe : ce qui pourrait sembler étonnant à première vue rejoint pourtant des réflexions contemporaines sur le rapprochement des artistes et des géographes, pour le partage de démarches de travail. C'est ce dont parle Anne Volvey dans « Entre l'art et la géographie, une question (d')esthétique ". L'article propose une synthèse d'un travail de recherche portant sur les emprunts et les chevauchements de pratiques entre artistes contemporains et chercheurs dans le cadre de travaux "de terrain", et interrogeant de ce fait la coupure épistémologique qui existe entre les arts et les sciences. Pour l'auteure, la pratique de certains artistes est à envisager comme une science de l'espace, tandis que la géographie peut s'entendre comme une esthétique de l'espace ${ }^{4}$. Cette réflexion est illustrée par plusieurs exemples révélateurs de ce croisement disciplinaire, et est mise en perspective théorique à travers le recours à la géographie postmoderne anglo-saxonne.

7 La rencontre des pratiques artistiques et scientifiques constitue un moyen d'analyse mais aussi d'action sur les territoires et/ou leurs représentations, à l'instar de ce que montrent Elsa Vivant, Burcu Ozdirlik et Nadia Arab dans «L'artiste, la carte et le territoire : détourner et retourner les représentations ». Les auteures croisent ici quatre cas d'utilisation de la cartographie participative où l'usage de la carte, outil d'expression privilégié du géographe, est détourné/réapproprié par les artistes. Tantôt intégrée à un spectacle de rue, envisagée comme une œuvre-enquête, vue comme un espace de rencontre et de sociabilité ou outil pour valoriser le territoire, la pratique cartographique appropriée par les artistes ne vise pas (uniquement) à faire œuvre, mais bien à contribuer aux réflexions sur les transformations territoriales en cours.

8 Le rôle des acteurs - artistes, professionnels de l'aménagement et habitants - relève d'un équilibre négocié pour chaque projet artistique : en travaillant sur la question de l'intégration d'œuvres d'art dans l'espace public, Martin Zebracki interroge le dialogue qui s'instaure entre les acteurs et la place singulière occupée par les experts dans les discussions («Public art as conversation piece: scaling art, public space 
and audience »). Construit autour de la participation de l'auteur à une session plénière d'experts de l'International Architecture Biennale Rotterdam, le texte témoigne des postures de recherche alternatives qui sont aujourd'hui intégrées par les géographes.

9 Son travail montre combien les décisions prises en matière d'art public dépendent des présupposés des experts et de la place qu'ils sont prêts à laisser aux publics dans les débats. Une fois l'œuvre négociée et installée dans l'espace, sa réception in situ est aussi révélatrice du rapport des sociétés à leurs espaces. Dans "L'art, un outil géographique pour mettre au jour et en œuvre la (dé)construction des espaces publics à Johannesburg (Afrique du Sud): le cas de Mandela Square ", Pauline Guinard montre ce que de telles œuvres font à l'espace public et aux passants. Travaillant sur une place de Johannesburg et sur différentes manifestations artistiques qui s'y sont déroulées, l'auteure utilise l'art « comme un prisme permettant de rendre visible et de distinguer (...) les différentes dimensions (juridique, sociale, politique) qui composent [les] espaces » (Guinard, §17).

10 Jannes van Loon écrit aussi à propos d'art placé dans l'espace public et dont l'existence, négociée selon des paramètres très différents toutefois, soulève des interrogations quant à sa réception. En traitant des graffiti et de leurs créateurs (les writers), le chercheur (ex-writer lui-même) envisage la production artistique sous l'angle du rapport des artistes à l'espace et donc de la spatialisation des graffiti. Dans « 'Just writing your name?' An analysis of the spatial behavior of graffiti writers in Amsterdam ", il montre que le comportement spatial des graffiti writers dépend d'un sentiment d'appartenance (sense of place) construit autour de leur appréhension de l'espace urbain et de ses cadres réglementaires.

11 A plus petite échelle, la perception et les représentations de l'espace urbain entrent également en ligne de compte dans la définition de «scènes » du rap à Paris et Atlanta. Celles-ci constituent le terrain d'investigation privilégié de Séverin Guillard, qui les étudie non pas à travers les représentations spatiales qu'elles véhiculent, mais à travers l'analyse des pratiques de leurs acteurs et du sens que ceux-ci attribuent aux événements artistiques auxquels ils participent. Son article, « 'To be in the place' : les open mics comme espaces de légitimation artistique pour les scènes rap à Paris et Atlanta ", montre comment se construisent deux modes de légitimation différenciés, propres à la façon dont se structurent les mondes du rap en France et aux États-Unis.

12 Paris et Atlanta constituent deux villes importantes pour la scène du rap; de manière plus générale, il a toujours existé dans l'histoire des arts des espaces hiérarchisés, polarisateurs des pratiques artistiques et servant de pôles de légitimation pour les créateurs et les interprètes. Au cours de sa carrière, quel plasticien ne rêve pas d'un jour exposer à New York, quel musicien de jouer dans une grande salle berlinoise ? Le passage par de tels lieux s'inscrit dans des carrières réputées pour être extrêmement mobiles : en dehors des monographies et des entretiens d'artistes, on sait cependant peu de choses des logiques et des formes prises par les mobilités d'artistes. Fabien Barthélémy et Camille Boichot soulèvent ici un coin du voile et remettent en perspective la figure de l'artiste nomade, décrivant « Entre mouvement et ancrages : les spatialités d'artistes mobiles ».

13 La question des hiérarchies spatiales, envisagée dans les deux derniers textes à échelle interurbaine, s'applique également à l'échelle intra-urbaine pour décrire la structuration des scènes artistiques. Les quatre contributions suivantes permettent de s'en rendre compte, abordant d'abord la question des quartiers d'artistes (à Cracovie et 
Katowice, New York et Pékin) puis celle des lieux de la production et de sa diffusion (à Bombay et Istanbul). C'est à travers l'exemple particulier des étudiants en art de deux villes polonaises que Jarosław Działek et Monika Murzyn-Kupisz se penchent sur les espaces privilégiés par les artistes, avec une dimension évolutive (" Young artists and the development of artistic quarters in Polish cities »). Mettant en évidence les similarités des processus liant la présence d'artistes et les phénomènes de gentrification avec ceux des villes occidentales, les deux auteurs livrent une analyse minutieuse basée sur un matériel empirique original, qui souligne par certains aspects le caractère exacerbé des transformations urbaines en Europe de l'Est.

Prenant un angle différent, Christian Morgner interroge quant à lui les logiques de production et d'évolution des quartiers artistiques à travers le concept de morphogénèse (" The morphogenesis of art districts: Case studies of Williamsburg, NYC and 798, Beijing "). L'auteur explicite son raisonnement théorique en l'illustrant avec deux cas d'étude contrastés (Williamsburg et le district 798 à Pékin). Ceux-ci révèlent des différences dans les facteurs inhérents au développement historique de ces espaces, mais ils offrent aussi à l'auteur l'opportunité de pointer des éléments récurrents servant de catalyseurs, telles que des infrastructures de mobilité, des institutions culturelles (répondant de la sorte à l'étude des villes polonaises), ou des éléments de nature plus symbolique. L'article insiste sur le fragile équilibre nécessaire à l'installation d'un quartier artistique et aux conditions qui rendent (im)probable sa permanence au fil du temps et des évolutions de son environnement.

En s'intéressant non pas aux quartiers d'artistes stricto sensu mais à tous les espaces de la production des œuvres d'art à Bombay, incluant aussi les acteurs invisibles du monde de l'art, Christine Ithurbide retrace le développement culturel historique de la ville, en insistant sur la nécessité de replacer l'art district au sein d'un réseau polycentrique, fonctionnant à plusieurs échelles spatiales: «Beyond Bombay art district: Reorganization of art production into a polycentric territory at metropolitan scale ". L'auteure décrit l'emboîtement des espaces, des plus informels aux plus légitimes, qui tous se transforment aujourd'hui sous l'influence des dynamiques urbaines locales mais aussi internationales.

La question de l'évolution des marchés de l'art traditionnels se trouve également au centre de l'analyse que livre Jérémie Molho du secteur des galeries d'art à Istanbul ( "Territorialisation d'un marché de l'art émergent : le cas d'Istanbul »). Alors que les acteurs privés locaux tentent de positionner la ville comme « hub artistique » vers le Moyen-Orient, il s'agit en même temps pour ces élites économiques et culturelles de promouvoir sur le marché international un certain exotisme de l'art contemporain turc. L'analyse des galeries stambouliotes traduit en réalité le développement de centralités différenciées de la part des deux marchés de l'art, contemporain et ottoman. Tous deux obéissent à des logiques spatiales et économiques distinctes, que voudraient toutefois rassembler artificiellement les acteurs locaux.

17 Le rôle des acteurs locaux dans la création d'une centralité culturelle au cœur des Territoires Palestiniens Occupés est l'objet de l'article collectif de Pauline Bosredon, Sophie Gravereau, Marie-Thérèse Grégoris et Anissa Habane, "Art et culture à Ramallah dans les Territoires palestiniens occupés (TPO): Entre patrimoine, revendications politiques et développement territorial ». Les auteures y étudient le développement culturel singulier de Ramallah, surnommé le "Paris de la Palestine » pour le nombre de ses équipements culturels. Portée par des artistes, des mécènes, des 
investisseurs et des bénévoles, la volonté de faire de Ramallah une nouvelle centralité culturelle s'inscrit dans un processus plus large de construction de l'image des Territoires palestiniens, de ses enjeux politiques et de développement territorial.

Bien qu'inscrits dans un tout autre contexte international, c'est aussi d'enjeux liés au redéveloppement territorial d'espaces délaissés dont il est question dans les deux derniers articles de ce numéro. A travers l'exemple étonnant du sambodrome de Rio de Janeiro, Laura Jouve-Villard montre comment se cristallisent les conflits sociaux de la société brésilienne autour d'un espace vide la majeure partie de l'année (et surnommé de fait "L'espace insulaire du carnaval des écoles de samba de Rio de Janeiro "). Structure gigantesque située à proximité d'un lieu qui rassemblait il y a un siècle les ancêtres des écoles de samba, auxquels il rend hommage, le sambodrome apparaît paradoxalement à l'échelle de son quartier comme une frontière urbaine, empêchant désormais les riverains de mener leur carnaval. Il semble aussi exclu des vastes programmes de revitalisation urbaine entamés à Rio avant d'accueillir les grandes manifestations sportives internationales (Coupe du Monde de football en 2014 et Jeux Olympiques en 2016).

Le dernier texte s'intitule "Les équipements culturels de la vallée de l'Emscher (Ruhr, Allemagne): de la régénération urbaine au développement d'une économique culturelle et créative » et est signé par Bruno Lusso. Il présente un cas emblématique de l'instrumentalisation à laquelle la culture peut être sujette à des fins de redéploiement économique local, en en dressant les caractéristiques et en saisissant tous les enjeux, les difficultés et les contradictions.

La grande variété des approches et des thématiques traitées au fil des quinze articles illustre au final à quel point l'art peut être une porte d'entrée féconde pour comprendre les dynamiques spatiales contemporaines. Comme le montrent les premiers articles de ce numéro, l'art comme démarche peut nourrir les réflexions sur l'espace et enrichir les pratiques et les représentations classiques en géographie, en aménagement ou en urbanisme pour une compréhension sensible et polyphonique des espaces. Pris comme objet, l'art, ou plus précisément les spatialités des mondes de l'art, sont considérés dans la majorité des articles comme un prisme pour comprendre les enjeux socio-spatiaux liés à la place et au rôle accordé à l'art et aux artistes aujourd'hui, et notamment dans les sociétés urbaines. Les différents textes rassemblés ici soulignent la diversité et la complexité des rapports entre art et espaces. Ils nous rappellent que l'art est aussi aujourd'hui utilisé voire instrumentalisé à des fins autres qu'artistiques, pour (re)créer de l'espace. Ils nous invitent en ce sens à une lecture critique des liens entre arts et espaces qui éclairent la place accordé à l'art et aux artistes dans les sociétés contemporaines.

Bonne lecture! 


\section{BIBLIOGRAPHIE}

BOICHOT C. (2014), « Les quartiers artistiques : territoires en construction. Regards croisés sur Montreuil (île-de-France) et Neukölln (Berlin) », Annales de Géographie, 698, pp. 1088-1111.

BOICHOT C. (2012), Centralités et territorialités artistiques dans la structuration des espaces urbains. Le cas de Paris et Berlin, Thèse de doctorat en géographie, Paris et Francfort-sur-l'Oder, Université Paris1 Panthéon Sorbonne et Université Viadrina.

CAQUARD S., PIATTI B., CARTWRIGHT W. (dir.) (2009), « Art \& Cartography », Special Issue of The Cartographic Journal, 46, 4.

DEBROUX T. (2014), « Boris Grésillon, Géographie de l'art. Ville et création artistique », Belgeo [En ligne], 1 | 2014, URL : http://belgeo.revues.org/12873.

DEBROUX T. (2013) « Les territoires créatifs : quelques notions théoriques et une analyse bruxelloise », Territoire en mouvement, 19-20, 2, pp. 40-59.

DEBROUX T. (2012), Des artistes en ville. Géographie rétrospective des plasticiens à Bruxelles (1833-2008), Thèse de doctorat en géographie, Bruxelles, Université Libre de Bruxelles.

GRÉSILLON B. (2014), Géographie de l'art. Ville et création artistique, Paris, Editions Economica. GUINARD P. (2014), Johannesburg. L'art d'inventer une ville, Rennes, Presses Universitaires de Rennes.

KAUFMANN DA COSTA Th. (2004), Toward a Geography of Art, Chicago, The University of Chicago Press.

MILES M. (2007), Cities and Culture, Londres et New York, Routledge.

MITCHELL D. (2000), Cultural Geography. A Critical Introduction, Oxford, Blackwell.

QUIROS K., IMHOFF A. (2014), Géo-esthétique, Parc St-Léger.

TERRIN J.-J. (dir.) (2012), La ville des créateurs, Marseille, Parenthèses.

VOLVEY A. (dir.) (2007), « Spatialités de l'Art », numéro thématique des Travaux de l'Institut de Géographie de Reims (TIGR), 129-130.

\section{NOTES}

1. Pour se faire une idée de la très grande diversité de la production scientifique reprise sous l'expression de "géographie culturelle", il est certainement intéressant de parcourir les sommaires de la revue Géographie et cultures, de Cultural Geographies ou Social \& Cultural Geography. Le terme de " géographie de la culture ", ou géographie de l'art, est lui plus restreint (Grésillon, 2014 ; Debroux, 2014), tout en faisant écho plus largement aux préoccupations de la géographie sociale et urbaine.

2. Depuis plusieurs années, chaque édition des conférences de l'Union Géographique Internationale (UGI), de la European Association of Geographical Societies (EUGEO), de l'Association of American Geographers (AAG) ou de la Royal Geographical Society britannique (RGS-IBG) comporte des sessions spécialisées sur les arts et l'espace. Pour ne citer que celles à venir prochainement, voir notamment «Urban geography of arts: the co-production of arts and cities » organisée lors de l'UGI Regional Conference 2015 par Pauline Guinard et Géraldine Molina (août 2015), ou « Art, spatial 
experimentation and knowledge production" initiée par Camille Boichot et Sarah Mekdjian à Budapest, lors de du $5^{\text {th }}$ EUGEO Congress on the Geography of Europe (août-septembre 2015).

3. «Spatialités de l'Art ", numéro 129-130 coordonné par Anne Volvey, Travaux de l'Institut de Géographie de Reims (TIGR), 2007.

4. Esthétique étant comprise ici comme la perception, l'appréhension de l'espace - voir note 1 de l'article d'Anne Volvey.

\section{AUTEURS}

\section{CAMILLE BOICHOT}

Géographie-Cités UMR 8504, Paris, France, camille.boichot@gmail.com

\section{TATIANA DEBROUX}

IGEAT / Laboratoire de Géographie humaine, Université Libre de Bruxelles, Belgique, tdebroux@ulb.ac.be

\section{BORIS GRÉSILLON}

UMR Telemme, Aix-en-Provence, France, boris.gresillon@free.fr 